\title{
Alterations of white matter fractional anisotropy in unmedicated obsessive-compulsive disorder
}

\author{
This article was published in the following Dove Press journal: \\ Neuropsychiatric Disease and Treatment \\ 28 December 2016 \\ Number of times this article has been viewed
}

\author{
Jiong Tao ${ }^{1,2}$ \\ Xianglan Wang ${ }^{2}$ \\ Zhiyong Zhong ${ }^{1,2}$ \\ Hongying $\mathrm{Han}^{2}$ \\ Sha Liu $^{3}$ \\ Shenglin Wen ${ }^{4}$ \\ Nianhong Guan ${ }^{2}$ \\ Lingjiang $\mathrm{Li}^{\prime}$
}

'Mental Health Institute of the Second

Xiangya Hospital, Central South

University, Changsha, ${ }^{2}$ Department

of Psychiatry, The Third Affiliated

Hospital of Sun Yat-sen University,

${ }^{3}$ Department of Radiology, Guangzhou

Huiai Hospital, Guangzhou,

${ }^{4}$ Department of Psychiatry, The

Fifth Affiliated Hospital of Sun Yat-

sen University, Zhuhai, People's

Republic of China
Correspondence: Lingjiang Li Mental Health Institute of the Second Xiangya Hospital, National Technology Institute of Psychiatry, Key Laboratory of Psychiatry and Mental Health of Hunan Province, Central South University, No 139 Renmin Middle Road, Changsha 4 I 00 I I, People's Republic of China Email Ilj2920@।63.com
Background: Abnormalities in white matter (WM) have previously been reported in patients with obsessive-compulsive disorder (OCD). However, there was some inconsistency in the results obtained for altered regions of WM. The aim of this study was to investigate fractional anisotropy (FA) in the WM of the whole brain in patients with OCD by using diffusion tensor imaging (DTI).

Methods: In total, 28 unmedicated patients with OCD and 28 healthy volunteers underwent DTI scan. A voxel-based analysis was used to compare FA values in WM of the two groups at a voxel threshold of $P<0.005$ with an extent threshold of $k>72$ voxels $(P<0.05$; Alphasim correction). Subsequently, correlation analysis was conducted in order to find the correlation between the mean FA values in significantly altered brain regions and Yale-Brown Obsessive Compulsive Scale (Y-BOCS) scores of the OCD patients.

Results: Compared with healthy volunteers, the OCD patients had lower FA value in the left lingual gyrus, right midbrain, and right precuneus. There were no regions with significantly higher FA values in OCD patients compared with healthy volunteers. The mean FA values in the above regions (left lingual, $r=0.019, P=0.923$; right midbrain, $r=-0.208, P=0.289$; and right precuneus, $r=-0.273, P=0.161$ ) had no significant correlation with the Y-BOCS scores of the OCD patients.

Conclusion: The findings of this study suggest that alterations in WM of the left lingual gyrus, right midbrain, and right precuneus are associated with the pathophysiology mechanism of OCD, and these microstructural alterations do not correlate with symptom severity of OCD.

Keywords: white matter, diffusion tensor imaging, fractional anisotropy, obsessive-compulsive disorder

\section{Background}

Obsessive-compulsive disorder (OCD) is characterized by recurrent obsessional thoughts and repetitive compulsive behaviors; however, the underlying neurobiological mechanism of OCD is still unknown. The structural and functional magnetic resonance imaging (MRI) techniques that have been developed in the past, more than 20 years, have made it possible to assess brain microstructure, and these techniques function noninvasively and explore the etiology of OCD. Most of the previous research studies have found altered activity within a cortico(fronto)-striato-thalamo-cortical circuitry in patients with OCD. ${ }^{1-5}$ Diffusion tensor imaging (DTI) is a commonly used imaging technique that quantifies the diffusion characteristics of water molecules in tissue and provides microstructural information about the brain white matter (WM) tracts. ${ }^{6}$ Fractional anisotropy (FA) is a commonly used parameter derived from DTI data. It is directionally dependent on water molecule diffusivity, quite sensitive to some of the tissue properties such as fiber density, axonal diameter, thickness of the myelin sheaths, 
and directionality of the fibers. Any reduction in WM FA reflects partly a change in tissue connectivity and integrity. ${ }^{\text {? }}$ Most DTI studies in patients with OCD applied voxel-based analysis (VBA) methods ${ }^{8}$ and tract-based spatial statistics (TBSS) to investigate changes in WM microstructure. VBA is a whole-brain analytic technique used to compare the FA differences between the groups. ${ }^{9}$

Most of the previous studies reported decreased FA in brains of patients with OCD. These involved brain regions mainly included the corpus callosum, the cingulate, and near regions. ${ }^{10}$ Bora et al found decreased FA in the body of the corpus callosum in patients with $\mathrm{OCD},{ }^{11}$ and Nakamae et al found decreased FA and increased radial diffusivity (RD) in the anterior corpus callosum. ${ }^{12}$ In addition to these regions, alternations of WM FA in other brain regions have been reported. Szeszko et al found significantly lower FA in the WM of bilateral anterior cingulate gyrus, bilateral supramarginal gyri, right posterior cingulate gyrus, and left lingual gyrus. ${ }^{13}$ Garibotto et al revealed that OCD patients showed significantly lower FA in the corpus callosum, cingulum, superior longitudinal fasciculus, and inferior fronto-occipital fasciculus bilaterally. ${ }^{14}$ Different from these findings, some other studies found an increased FA value in OCD patients. For instance, Yoo et al studied 13 unmedicated OCD patients and found significantly increased FA in the corpus callosum, the internal capsule, and WM in the region superolateral to the right caudate. ${ }^{15}$ Apart from the findings of decreased or increased FA in many studies, some other studies found both decreased and increased FA in the same group of patients with OCD. For instance, a study by Lochner et al showed that patients with OCD had increased FA in bilateral regions of the anterior limb of the internal capsule (ALIC) adjacent to the body of the caudate, as well as decreased FA in the right anterior limb near the head of the caudate. ${ }^{16}$ Menzies et al studied the WM alternations of 30 patients with OCD and their first-degree relatives applying VBA methods. Both OCD patients and their relatives showed significantly decreased FA in the right inferior parietal lobe and significantly increased FA in the right medial frontal cortex. ${ }^{17}$

Some researchers found the alterations of FA in WM, and then they evaluated the association between the FA value and the clinical symptom severity. ${ }^{14}$ Koch et al investigated the association between symptom dimensions and WM microstructure and found that the ordering dimension was negatively correlated with FA in the right inferior frontooccipital fasciculus and the right optic radiation. ${ }^{18}$ Saito et al found that higher FA in the rostrum was correlated significantly with lower Yale-Brown Obsessive Compulsive
Scale (Y-BOCS) score. ${ }^{10}$ Szeszko et al found that lower FA in the parietal region was significantly correlated with higher Y-BOCS scores. ${ }^{13}$ Some other studies did not report whether the alterations of WM FA were correlated with the clinical symptom severity.

In general, previous studies have investigated the underlying imaging mechanisms of OCD and reported alterations in WM FA in patients with OCD; however, the results are inconsistent. The present study hypothesized that patients with OCD would exhibit more widespread WM abnormalities in addition to the previously reported findings. Thus, the purpose of this study was to investigate the WM abnormalities in patients with OCD by using DTI and VBA. The present study compared the WM FA in the whole brain between patients with OCD and healthy control (HC) subjects and then investigated the correlation between the mean FA in the significant brain regions and the Y-BOCS scores of patients with OCD.

\section{Methods}

\section{Subject and OCD symptom assessment}

This study compared 28 unmedicated OCD patients with $28 \mathrm{HC}$ subjects. All the patients were recruited by a senior psychiatrist from the outpatients of Department of Psychiatry in the Third Affiliated Hospital of Sun Yat-sen University, Guangzhou, China, who met the Diagnostic and Statistical Manual of Mental Disorders 1994 (DSM-IV) criteria for OCD, with exclusion of other DSM-IV Axis I disorders (American Psychiatric Association, 1994). All the participants were Han Chinese and right-handed and had no history of neurological disease. Initially, 34 patients with OCD and 29 healthy volunteers were recruited, of which six patients and one healthy subject were excluded later because of some abnormal findings (such as cyst in cisterna magna and WM degeneration in frontal lobe) on subsequent MRI examinations. The participants in the two groups were matched for age, sex, and education.

During a clinical interview, the participants were assessed with the Mini-International Neuropsychiatric Interview, for $D S M-I V$ Axis I mental disorders, and the Y-BOCS, for OCD symptom and severity.

\section{MRI data acquisition}

All the image data were acquired by using a 3.0-T Philips Achieva MRI scanner (PHILIPS Achieva X-series; Philips Medical, Best, the Netherlands) in Huiai Hospital, Guangzhou, China. High-resolution three-dimensional (3D) T1-weighted imaging was conducted by using a fast-field echo-3D T1 sequence (repetition time $[\mathrm{TR}]=8 \mathrm{~ms}$, echo 
time $[\mathrm{TE}]=4 \mathrm{~ms}$, slice thickness $=1.0 \mathrm{~mm}$, flip angle $=7^{\circ}$, and voxel size $=1 \times 1 \times 1 \mathrm{~mm}^{3}$ ) before the DTI scan to exclude the subjects with brain abnormalities.

A single-shot, spin echo-echo planar imaging sequence $(\mathrm{TR}=8,500 \mathrm{~ms}$, TE $=70 \mathrm{~ms}$, matrix $=128 \times 128$, field of view $=256 \times 256 \mathrm{~mm}$, slice thickness $=2.0 \mathrm{~mm}$, slice gap $=0$, voxel size $=2 \times 2 \times 2 \mathrm{~mm}^{3}$, and flip angle $=90^{\circ}$ ) was used to acquire the DTI images. Diffusion sensitizing gradients were applied along 33 noncollinear directions $\left(b=1,000 \mathrm{~s} / \mathrm{mm}^{2}\right)$ and one $b_{0}$ volume without diffusion gradient $(b=0)$.

\section{MRI data processing}

All the data were processed by using Statistical Parametric Mapping (SPM8; http://www.fil.ion.ucl.ac.uk/spm/) and software tools from the Functional MRI of the Brain (FMRIB) software library FSL (FSL, http://www.fmrib.ox.ac.uk/fsl/). First, the diffusion data set was prealigned to correct for head motion and the effects of gradient coil eddy currents using the eddy correct toolbox in FSL. ${ }^{19}$ Then, the diffusion tensor at each voxel was calculated by using the FMRIB diffusion toolbox in FSL. The resulting FA images were transformed into Montreal Neurological Institute (MNI) standard space with SPM8 (Wellcome Department of Cognitive Neurology, London, UK) by means of the following steps: the $b=0$ images were coregistered with the T1 image for that individual, the same coregistration parameters were applied to the FA maps (in the same space as the $b=0$ images), each individual's T1 image was then normalized to the SPM T1 template in MNI standard space, and the same normalization parameters were then applied to the coregistered FA images. Subsequently, FA images were smoothened with a 6-mm full width at half maximum Gaussian kernel in order to decrease spatial noise and compensate for the inexact nature of normalization. Finally, all the images were resampled with a voxel size of $2 \times 2 \times 2 \mathrm{~mm}^{3}$. In order to use in the two-sample $t$-test, a binary WM mask was made by calculating a mean FA image with a threshold FA value of 0.25 from FA images of all the participants. ${ }^{20}$

\section{Statistical analysis}

A whole-brain VBA was performed by using SPM8 software, which had been frequently used in the previous studies on OCD. The normalized and smoothed FA maps were compared between the OCD and HC groups by two-sample group $t$-tests. A WM mask made while processing was used to restrict the region for analysis. ${ }^{8}$

The statistical result was corrected for multiple comparisons by using the "AlphaSim" implementation in the Resting-State fMRI Data Analysis Toolkit (REST). ${ }^{21}$ This function is based on the Monte Carlo simulation in Analysis of Functional NeuroImages (AFNI) (see the AlphaSim command description at http://afni.nimh.nih.gov/afni/doc/manual/ AlphaSim).

The statistical threshold for each voxel was set at $P<0.005$ with a cluster size $k>72$ voxels, which yielded a corrected $P<0.05$ (AlphaSim program). Then, the corresponding two samples $t$-test in SPM8 results were superimposed onto the averaged maps, by using xjview (http://www.alivelearn.net/ xjview8).

In order to investigate the clinical association of significant clusters further, the mean FA value of each region of interest (ROI) was extracted from the FA maps of every subject ${ }^{20}$ in the OCD group by using the utility for ROI time-course extraction in the REST. ${ }^{21}$ The ROI mask was constructed by setting the peak coordinate of each significant cluster as the center of a sphere with the radius $=6 \mathrm{~mm}$, respectively.

\section{Ethics statement}

The research protocol was approved by the Ethics Committee of the Second Xiangya Hospital of Central South University. Written informed consent was obtained from all the participants.

\section{Results \\ Demographic characteristics and clinical data}

Table 1 shows the demographic characteristics and clinical data of the two groups. The OCD group had a mean Y-BOCS total score of 21.75 (standard deviation $[\mathrm{SD}]=5.17$ ), and the HC group had a mean Y-BOCS total score of $4.79(\mathrm{SD}=1.95$, $P<0.001)$. There were no significant differences in the demographic data such as age, sex, and education level between the two groups $(P>0.05)$.

\section{Group comparison of FA}

Significant FA reductions were found in the OCD group, compared with controls, in the left lingual gyrus, right midbrain, and right precuneus. Table 2 lists the significant clusters, and Figure 1 shows a map of decreased FA. There were no clusters with significantly higher FA in OCD patients compared with healthy volunteers.

\section{Correlation analysis between symptom severity and FA}

No significant correlation was found between Y-BOCS scores and mean FA values of altered brain regions (lingual_L, $r=0.019, P=0.923$; midbrain, $r=-0.208, P=0.289$; and precuneus, $r=-0.273, P=0.161$ ) in the OCD patients. Figure 2 
Table I The demographics and clinical characteristics of the participants

\begin{tabular}{|c|c|c|c|}
\hline Variables & $\operatorname{OCD}(n=28)$ & $H C(n=28)$ & $\begin{array}{l}\text { Group } \\
\text { comparison }(P)\end{array}$ \\
\hline Age (years, mean $\pm S D$ ) & $25.62 \pm 10.54$ & $27.39 \pm 8.63$ & $0.49 I^{\mathrm{a}}$ \\
\hline Sex (number, male/female) & $16 / 12$ & $13 / 15$ & $0.422^{\mathrm{b}}$ \\
\hline Education (years, mean \pm SD) & 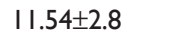 & $12.62 \pm 2.61$ & $0.132^{\mathrm{a}}$ \\
\hline Y-BOCS score (mean \pm SD) & $21.75 \pm 5.17$ & $4.79 \pm 1.95$ & $0.000^{\mathrm{a}}$ \\
\hline
\end{tabular}

Notes: andependent-Samples $t$-test in SPSS, ${ }^{b}$ chi-square test.

Abbreviations: HC, healthy control; OCD, obsessive-compulsive disorder; SD, standard deviation; Y-BOCS, Yale-Brown Obsessive Compulsive Scale.

Table 2 The FA values of different brain regions between the OCD and HC groups

\begin{tabular}{lllll}
\hline Different regions & Hemisphere & Cluster size & $\begin{array}{l}\text { Peak } \\
\text { coordinates }(x, y, z)\end{array}$ & $T$-value \\
\hline OCD $<$ HC & & & & 3.611 \\
Midbrain & $\mathrm{R}$ & 72 & $10,-24,-10$ & 3.454 \\
Lingual gyrus & $\mathrm{L}$ & 74 & $-12,-90,-8$ & 3.499 \\
Precuneus & $\mathrm{R}$ & 139 & $22,-54,24$ & \\
\hline
\end{tabular}

Notes: aSignificant threshold: $d f=54, P<0.005(T=2.670)$ with $k>72$ voxels $(P<0.05$, corrected by AlphaSim correction). Peak coordinates represent the location of the maximum pixel values in standard Montreal Neurological Institute space.

Abbreviations: FA, fractional anisotropy; $\mathrm{HC}$, healthy control; L, left; OCD, obsessive-compulsive disorder; R, right.

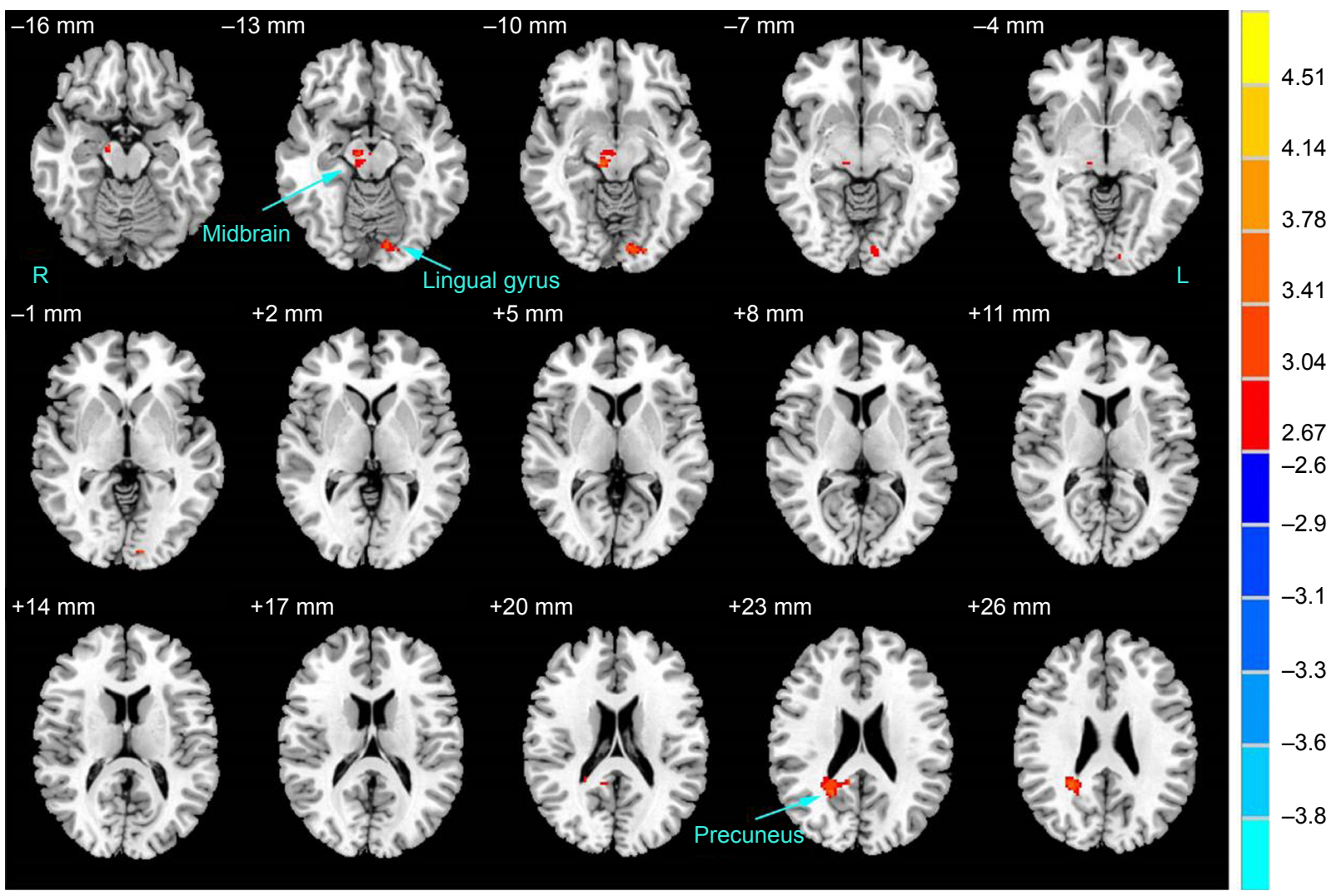

Figure I Brain regions with altered FA in subjects with $\mathrm{OCD}$ compared to HCs.

Notes: A significant height threshold of $P<0.005$ with an extent threshold of $k>72$ voxels $(P<0.005$, corrected by AlphaSim correction) was set for the two-sample $t$-test. Warm colors represent decreased FA values $(\mathrm{OCD}<\mathrm{HC})$. The details of altered regions are listed in Table 2.

Abbreviations: FA, fractional anisotropy; HC, healthy control; L, left; $\mathrm{OCD}$, obsessive-compulsive disorder; R, right. 

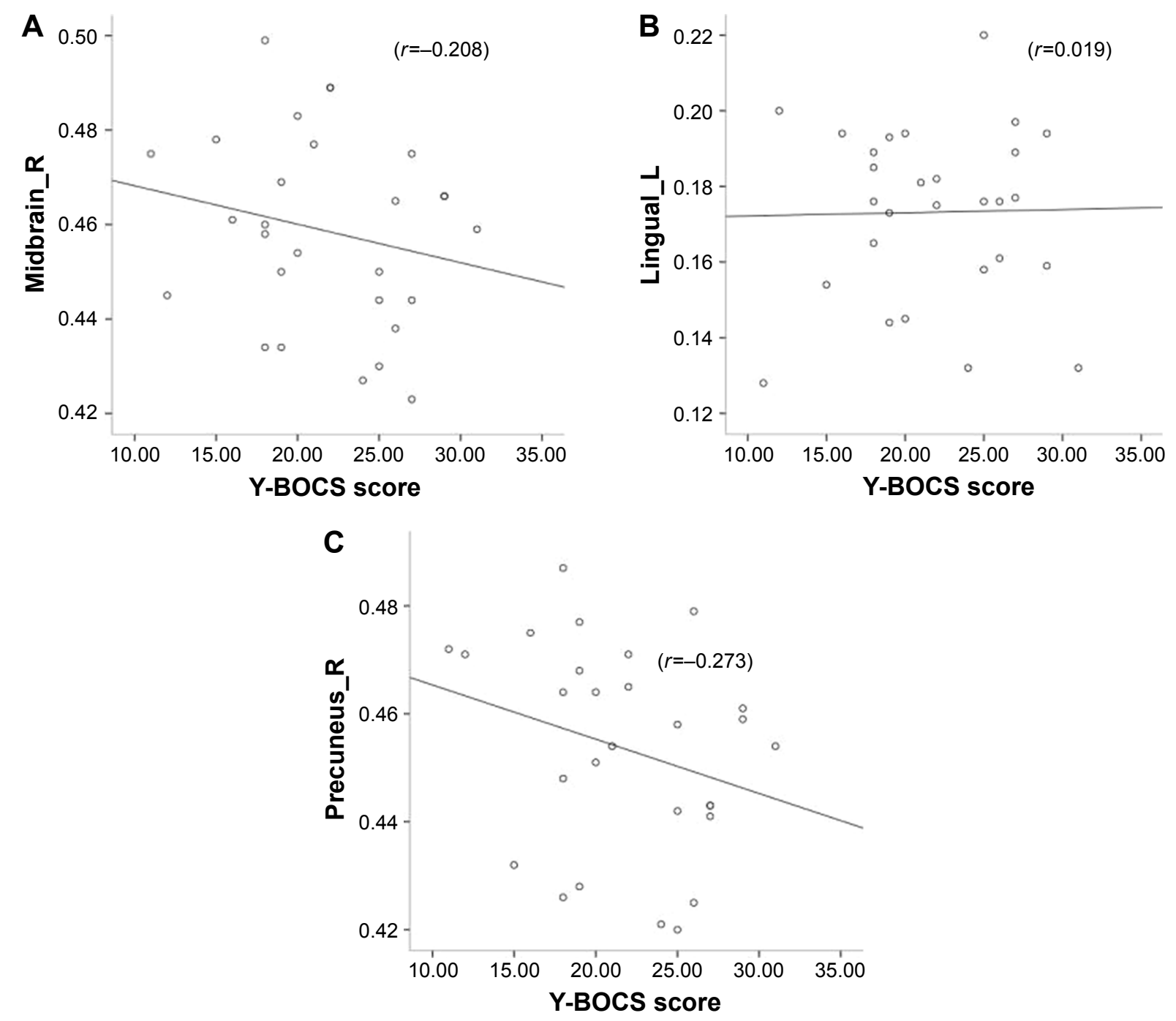

Figure 2 Scatterplots between mean FA values in altered brain regions and $\mathrm{Y}$-BOCS score.

Notes: (A) Correlation between FA values in the right midbrain and Y-BOCS score; (B) correlation between FA values in the lingual gyrus and Y-BOCS score; (C) correlation between FA values in the right precuneus and Y-BOCS score.

Abbreviations: FA, fractional anisotropy; Y-BOCS, Yale-Brown Obsessive Compulsive Scale.

shows the scatterplots between mean FA values in altered brain regions and Y-BOCS scores.

\section{Discussion}

This study investigated WM abnormalities of unmedicated patients with OCD by using DTI methods. The main findings are the decreased FA values in the left lingual gyrus, right midbrain, and right precuneus WM of OCD patients compared with $\mathrm{HC}$, and the mean $\mathrm{FA}$ values in the above regions had no significant correlation with the Y-BOCS scores of the OCD patients. Different from previous studies, ${ }^{1-5}$ the findings from the present study revealed that WM alterations in new brain regions may play a role in the development of OCD.

Lowered FA means decreased WM connectivity and has been considered to be indicative of WM microstructural abnormalities. ${ }^{6}$ In general, the findings of voxel-based DTI studies in OCD patients are still rather heterogeneous. ${ }^{7}$ Most of the previous studies reported decreased FA values in OCD patients compared to healthy subjects, and the corpus callosum and the cingulate and adjacent areas were mostly involved. ${ }^{10,11}$ Nakamae et al found decreased FA and increased RD in the corpus callosum. ${ }^{12}$ Szeszko et al found lower FA in the bilateral anterior cingulate gyrus WM, bilateral supramarginal gyri, right posterior cingulate gyrus, and left lingual gyrus. ${ }^{13}$ Garibotto et al revealed lower FA in the corpus callosum, cingulum, superior longitudinal fasciculus, and inferior fronto-occipital fasciculus bilaterally. ${ }^{14}$ Although most of the DTI studies reported lower FA values, there are also a number of studies that found increased FA in OCD patients. Li et al studied partly medicated OCD patients and 
reported significantly increased FA in the corpus callosum (genu and body) as well as in the WM of the right superior frontal gyrus. ${ }^{22}$ Nakamae et al found increased FA in the bilateral semioval center extending to the subinsular WM. ${ }^{23}$ Yoo et al reported increased FA in corpus callosum, internal capsule, and WM in the area superolateral to the right caudate. ${ }^{15}$ Few studies reported increased FA in some brain regions and decreased FA in other brain regions. Lochner et al found decreased FA in the bilateral ALIC adjacent to the body of the caudate and in the right anterior limb near the head of the caudate. ${ }^{16}$ Possible reasons for inconsistent results are the heterogeneity of sample size, age of patients, OCD symptom dimensions, and disease stages. Further research is required to clarify WM alterations in OCD patients.

The present study found lower FA values in the left lingual gyrus WM of OCD patients compared with HCs. There were few reports about WM abnormalities in the lingual gyrus. Szeszko et al revealed lower FA bilaterally in the parietal region (supramarginal gyri), right posterior cingulate gyrus, and left occipital lobe (lingual gyrus). ${ }^{13}$ Although the lingual gyrus is known to play a role in mediating the specific visual word processing and/or general analysis of complex graphical features of visual forms, ${ }^{24}$ some data suggest that it has more features..$^{25,26}$ This region may be thought to be involved in selective attention. ${ }^{27,28}$ Selective attention allows to focus on relevant information and to ignore distracting features of a visual scene. ${ }^{29}$ Previous studies have indicated that selective attention deficit is especially relevant for OCD. ${ }^{30}$ There was a significant deterioration in performance on selective attention tasks for the OCD patients. ${ }^{31} \mathrm{~A}$ diminished ability of OCD patients to selectively ignore competing stimuli, especially intrusive thoughts, was observed. ${ }^{32}$ Hence, it was assumed that alterations of WM microstructure in the lingual gyrus that are involved in selective attention deficits underlie the development of OCD.

Lower FA values were found in the right midbrain WM of OCD patients compared with HCs. Previously, only a few studies using DTI methods reported midbrain abnormalities in OCD patients. Alexopoulos et al found lower FA in the neostriatum and midbrain as well as selected temporal and parietal regions. ${ }^{33}$ Fan et al found an increased RD in right midbrain of OCD patients. ${ }^{8}$ Midbrain is connected to limbic area (ie, amygdala), ${ }^{34}$ and the alternations of WM microstructure in midbrain may affect nerve conduction between midbrain and limbic area that is involved in emotional adjustment. Some studies reported abnormalities in midbrain of OCD patients by using other methods. Gilbert et al found significant gray matter volumetric differences in bilateral midbrain of OCD patients relative to controls and revealed positive correlation between OCD symptom severity and gray matter volumes in bilateral midbrain. ${ }^{35}$ Saxena et al found higher midbrain glucose metabolism in nonhoarding OCD patients relative to controls. ${ }^{36} \mathrm{~A}$ number of studies reported abnormalities in serotonin receptors in midbrains of OCD patients. ${ }^{37-39}$ Reduced serotonin transporter availability in midbrain supports the serotonin deficit hypothesis of OCD. Thus, it can be assumed that WM alternations in midbrain as well as abnormalities of gray matter and serotonin receptors together constitute the pathophysiological mechanisms underlying the development of OCD.

Lower FA values were found in the right precuneus WM of OCD patients compared with HCs. Few reports are found on WM abnormalities in the right precuneus in OCD patients. Yagi et al revealed positive correlation between the severity of ordering symptom in OCD patients and FA value in right precuneus and negative correlations with $\mathrm{RD}$ value in right precuneus. ${ }^{40}$ Many studies have found abnormalities in precuneus gray matter in OCD patients. ${ }^{17,41,42}$ The precuneus receives rich input from the thalamic pulvinar nuclei and the left medial temporal cortex. ${ }^{43}$ The changes in WM microstructure in the precuneus may affect information transmission in these regions. Jones and Bhattacharya indicated that the precuneus plays a role in thought-action fusion (TAF) in OCD. ${ }^{44}$ TAF is a cognitive bias assumed to underlie the development of OCD, and negative emotion mediates the relationship between TAF and OCD. ${ }^{45}$ Alternations of WM microstructure in the right precuneus in OCD patients may be related to TAF and then contribute to the development of OCD. In contrast, previous studies have found that TAF is also related to other anxiety disorders; ${ }^{45}$ therefore, further studies are required to clarify it.

In the previous studies, the FA values in the left lingual gyrus, right midbrain, and right precuneus have no significant correlation with the Y-BOCS scores of the OCD patients, respectively. A few previous studies have found the existence of correlation between symptom severity and WM changes; ${ }^{18,22,40}$ Yagi et al revealed a positive correlation between ordering severity and FA value in right precuneus and a negative correlations between ordering severity and RD value in right precuneus in OCD patients and indicated that different OCD symptom dimensions were associated with different patterns of WM abnormalities. ${ }^{40}$ As mentioned above, inconsistent results from previous studies were partly due to heterogeneity of sample size, age of patients, OCD symptom dimensions, and disease stages. Image acquisition and data analysis were partly responsible for the heterogeneity of results. Ha et al indicated that OCD may be a heterogeneous 
disease with distinct WM changes, ${ }^{46}$ which suggests that the correlation between WM alternations and symptom dimensions in OCD patients remains to be clarified.

\section{Limitations}

The present study has several limitations. First, the sample size was relatively small for analyzing the correlation between OCD symptom severity (Y-BOCS total score) and FA values. Second, limited by the sample size, the alternations of WM FA values were not investigated in patients with different OCD symptom dimensions. Third, a cross-sectional study was adopted and did not tract the change before and after treatment. Therefore, future research with a larger sample size of participants with a variety of symptoms is required. In addition, other analytic methods for the WM microstructure in the brain, such as TBSS, offer more details about the underlying brain mechanism of the OCD.

\section{Conclusion}

The findings from the present study suggest that WM alterations in left lingual gyrus, right midbrain, and right precuneus may play a role in the pathophysiological mechanism of OCD, and the microstructural alterations in these regions are not correlated with symptom severity.

\section{Acknowledgments}

The authors thank Prof Guimao Huang from Department of Radiology of Guangzhou Huiai Hospital for his strong support on MRI scanning and acknowledge support by grants from the National Natural Science Foundation of China (81171286 and 91232714 to Lingjiang Li), National Basic Research Program of China (2013CB835100 and 2009CB918303 to Lingjiang Li), National Science and Technologic Program of China (2015BAI13B02 to Lingjiang Li), and the Science and Technology Program of Guangdong, China (2012B031800357 to Hongying Han).

\section{Author contributions}

Lingjiang Li and Jiong Tao designed the study and wrote the protocol. Jiong Tao managed the literature searches and analyses and wrote the first draft of this manuscript. Jiong Tao and Xianglan Wang undertook the MRI data processing and the statistical analysis. All authors contributed toward data analysis, drafting and revising the paper and agree to be accountable for all aspects of the work.

\section{Disclosure}

The authors report no conflicts of interest in this work.

\section{References}

1. Ting JT, Feng G. Neurobiology of obsessive-compulsive disorder: insights into neural circuitry dysfunction through mouse genetics. Curr Opin Neurobiol. 2011;21(6):842-848.

2. Piras F, Piras F, Caltagirone C, Spalletta G. Brain circuitries of obsessive compulsive disorder: a systematic review and meta-analysis of diffusion tensor imaging studies. Neurosci Biobehav Rev. 2013; 37(10 Pt 2):2856-2877.

3. Peng Z, Lui SS, Cheung EF, et al. Brain structural abnormalities in obsessive-compulsive disorder: converging evidence from white matter and grey matter. Asian J Psychiat. 2012;5(4):290-296.

4. Eng GK, Sim K, Chen SH. Meta-analytic investigations of structural grey matter, executive domain-related functional activations, and white matter diffusivity in obsessive compulsive disorder: an integrative review. Neurosci Biobehav Rev. 2015;52:233-257.

5. Radua J, Grau M, van den Heuvel OA, et al. Multimodal voxel-based meta-analysis of white matter abnormalities in obsessive-compulsive disorder. Neuropsychopharmacology. 2014;39(7):1547-1557.

6. Le Bihan D, Mangin JF, Poupon C, et al. Diffusion tensor imaging: concepts and applications. J Magn Reson Imaging. 2001;13(4):534-546.

7. Koch K, Reess TJ, Rus OG, Zimmer C, Zaudig M. Diffusion tensor imaging (DTI) studies in patients with obsessive-compulsive disorder (OCD): a review. J Psychiatr Res. 2014;54:26-35.

8. Fan Q, Yan X, Wang J, et al. Abnormalities of white matter microstructure in unmedicated obsessive-compulsive disorder and changes after medication. PLoS One. 2012;7(4):e35889.

9. Thomason ME, Thompson PM. Diffusion imaging, white matter, and psychopathology. Ann Rev Clin Psychol. 2011;7:63-85.

10. Saito Y, Nobuhara K, Okugawa G, et al. Corpus callosum in patients with obsessive-compulsive disorder: diffusion-tensor imaging study. Radiology. 2008;246(2):536-542.

11. Bora E, Harrison BJ, Fornito A, et al. White matter microstructure in patients with obsessive-compulsive disorder.JPsychiatry Neurosci. 2011; 36(1):42-46.

12. Nakamae T, Narumoto J, Sakai Y, et al. Diffusion tensor imaging and tract-based spatial statistics in obsessive-compulsive disorder. J Psychiatr Res. 2011;45(5):687-690.

13. Szeszko PR, Ardekani BA, Ashtari M, et al. White matter abnormalities in obsessive-compulsive disorder: a diffusion tensor imaging study. Arch Gen Psychiat. 2005;62(7):782-790.

14. Garibotto V, Scifo P, Gorini A, et al. Disorganization of anatomical connectivity in obsessive compulsive disorder: a multi-parameter diffusion tensor imaging study in a subpopulation of patients. Neurobiol Dis. 2010;37(2):468-476.

15. Yoo SY, Jang JH, Shin YW, et al. White matter abnormalities in drugnaive patients with obsessive-compulsive disorder: a diffusion tensor study before and after citalopram treatment. Acta Psychiatr Scand. 2007; 116(3):211-219.

16. Lochner C, Fouche JP, du Plessis S, et al. Evidence for fractional anisotropy and mean diffusivity white matter abnormalities in the internal capsule and cingulum in patients with obsessive-compulsive disorder. J Psychiatry Neurosci. 2012;37(3):193-199.

17. Menzies L, Williams GB, Chamberlain SR, et al. White matter abnormalities in patients with obsessive-compulsive disorder and their firstdegree relatives. Am J Psychiatry. 2008;165(10):1308-1315.

18. Koch K, Wagner G, Schachtzabel C, et al. White matter structure and symptom dimensions in obsessive-compulsive disorder. $J$ Psychiatr Res. 2012;46(2):264-270.

19. Liao Y, Tang J, Ma M, et al. Frontal white matter abnormalities following chronic ketamine use: a diffusion tensor imaging study. Brain. 2010;133(Pt 7):2115-2122.

20. Wang XL, Tao J, Li LJ, et al. Alterations in white matter fractional anisotropy in subsyndromal perimenopausal depression. $B M C$ Psychiatry. 2014;14:367.

21. Song XW, Dong ZY, Long XY, et al. REST: a toolkit for resting-state functional magnetic resonance imaging data processing. PLoS One. 2011;6(9):e25031. 
22. Li F, Huang X, Yang Y, et al. Microstructural brain abnormalities in patients with obsessive-compulsive disorder: diffusion-tensor MR imaging study at 3.0 T. Radiology. 2011;260(1):216-223.

23. Nakamae T, Narumoto J, Shibata K, et al. Alteration of fractional anisotropy and apparent diffusion coefficient in obsessive-compulsive disorder: a diffusion tensor imaging study. Prog Neuropsychopharmacol Biol Psychiatry. 2008;32(5):1221-1226.

24. Kuriki S, Takeuchi F, Hirata Y. Neural processing of words in the human extrastriate visual cortex. Brain Res Cogn Brain Res. 1998;6(3):193-203.

25. Lang PJ, Bradley MM, Fitzsimmons JR, et al. Emotional arousal and activation of the visual cortex: an fMRI analysis. Psychophysiology. 1998;35(2):199-210.

26. Lane RD, Chua PM, Dolan RJ. Common effects of emotional valence, arousal and attention on neural activation during visual processing of pictures. Neuropsychologia. 1999;37(9):989-997.

27. Carter CS, Mintun M, Cohen JD. Interference and facilitation effects during selective attention: an H215O PET study of Stroop task performance. Neuroimage. 1995;2(4):264-272.

28. Allison T, Begleiter A, McCarthy G, Roessler E, Nobre AC, Spencer DD. Electrophysiological studies of color processing in human visual cortex. Electroencephalogr Clin Neurophysiol. 1993;88(5): 343-355.

29. Harnack D, Ernst UA, Pawelzik KR. A model for attentional information routing through coherence predicts biased competition and multistable perception. J Neurophysiol. 2015;114(3):1593-1605.

30. Koch J, Exner C. Selective attention deficits in obsessive-compulsive disorder: the role of metacognitive processes. Psychiatry Res. 2015; 225(3):550-555.

31. Cohen Y, Lachenmeyer JR, Springer C. Anxiety and selective attention in obsessive-compulsive disorder. Behav Res Ther. 2003;41(11): 1311-1323.

32. Clayton IC, Richards JC, Edwards CJ. Selective attention in obsessivecompulsive disorder. J Abnorm Psychol. 1999;108(1):171-175.

33. Alexopoulos GS, Murphy CF, Gunning-Dixon FM, et al. Microstructural white matter abnormalities and remission of geriatric depression. Am J Psychiatry. 2008;165(2):238-244.

34. Etkin A, Prater KE, Schatzberg AF, Menon V, Greicius MD. Disrupted amygdalar subregion functional connectivity and evidence of a compensatory network in generalized anxiety disorder. Arch Gen Psychiatry. 2009;66(12):1361-1372.
35. Gilbert AR, Mataix-Cols D, Almeida JR, et al. Brain structure and symptom dimension relationships in obsessive-compulsive disorder: a voxelbased morphometry study. J Affect Disord. 2008;109(1-2):117-126.

36. Saxena S, Brody AL, Maidment KM, et al. Cerebral glucose metabolism in obsessive-compulsive hoarding. Am J Psychiatry. 2004;161(6): 1038-1048.

37. Hesse S, Muller U, Lincke T, et al. Serotonin and dopamine transporter imaging in patients with obsessive-compulsive disorder. Psychiatry Res. 2005;140(1):63-72.

38. Stengler-Wenzke K, Muller U, Angermeyer MC, Sabri O, Hesse S. Reduced serotonin transporter-availability in obsessive-compulsive disorder (OCD). Eur Arch Psychiatry Clin Neurosci. 2004;254(4): 252-255.

39. Reimold M, Smolka MN, Zimmer A, et al. Reduced availability of serotonin transporters in obsessive-compulsive disorder correlates with symptom severity - a [11C]DASB PET study. J Neural Transm (Vienna). 2007;114(12):1603-1609.

40. Yagi M, Hirano Y, Nakazato M, et al. Relationship between symptom dimensions and white matter alterations in obsessive-compulsive disorder. Acta Neuropsychiatr. 2016;Sep 13:1-11.

41. Soriano-Mas C, Pujol J, Alonso P, et al. Identifying patients with obsessive-compulsive disorder using whole-brain anatomy. Neuroimage. 2007;35(3):1028-1037.

42. Rotge JY, Guehl D, Dilharreguy B, et al. Provocation of obsessivecompulsive symptoms: a quantitative voxel-based meta-analysis of functional neuroimaging studies. J Psychiatry Neurosci. 2008;33(5): 405-412.

43. Shaw P, Sharp W, Sudre G, et al. Subcortical and cortical morphological anomalies as an endophenotype in obsessive-compulsive disorder. Mol Psychiatry. 2015;20(2):224-231.

44. Jones R, Bhattacharya J. A role for the precuneus in thought-action fusion: evidence from participants with significant obsessive-compulsive symptoms. Neuroimage Clin. 2014;4:112-121.

45. Abramowitz JS, Whiteside S, Lynman D, Kalsy S. Is thought-action fusion specific to obsessive-compulsive disorder?: a mediating role of negative affect. Behav Res Ther. 2003;41(9):1069-1079.

46. Ha TH, Kang DH, Park JS, et al. White matter alterations in male patients with obsessive-compulsive disorder. Neuroreport. 2009; 20(7):735-739
Neuropsychiatric Disease and Treatment

\section{Publish your work in this journal}

Neuropsychiatric Disease and Treatment is an international, peerreviewed journal of clinical therapeutics and pharmacology focusing on concise rapid reporting of clinical or pre-clinical studies on a range of neuropsychiatric and neurological disorders. This journal is indexed on PubMed Central, the 'PsycINFO' database and CAS,

\section{Dovepress}

and is the official journal of The International Neuropsychiatric Association (INA). The manuscript management system is completely online and includes a very quick and fair peer-review system, which is all easy to use. Visit http://www.dovepress.com/testimonials.php to read real quotes from published authors. 